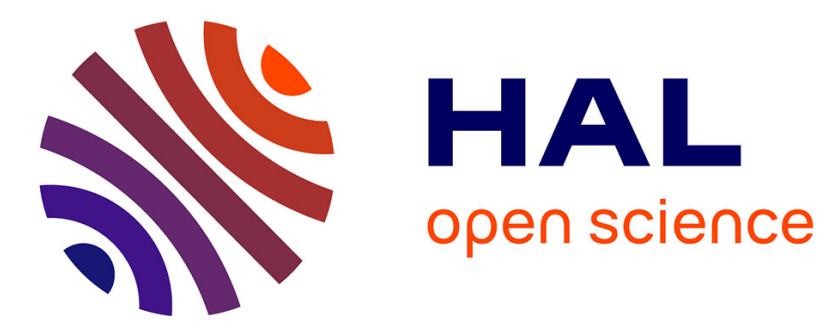

\title{
Sasaguri. Dedans, dehors : une approche ethnologique collective
}

\author{
Anne Bouchy
}

\section{To cite this version:}

Anne Bouchy. Sasaguri. Dedans, dehors: une approche ethnologique collective. Cahiers d'ExtrêmeAsie, 2013, no. 22, p. 1-25. 10.3406/asie.2013.1414 . halshs-03134678

\section{HAL Id: halshs-03134678 \\ https://shs.hal.science/halshs-03134678}

Submitted on 8 Feb 2021

HAL is a multi-disciplinary open access archive for the deposit and dissemination of scientific research documents, whether they are published or not. The documents may come from teaching and research institutions in France or abroad, or from public or private research centers.
L'archive ouverte pluridisciplinaire HAL, est destinée au dépôt et à la diffusion de documents scientifiques de niveau recherche, publiés ou non, émanant des établissements d'enseignement et de recherche français ou étrangers, des laboratoires publics ou privés. 


\section{Sasaguri. Dedans, dehors : une approche ethnologique collective} Anne Bouchy

\section{Citer ce document / Cite this document :}

Bouchy Anne. Sasaguri. Dedans, dehors : une approche ethnologique collective. In: Cahiers d'Extrême-Asie, vol. 22, 2013. Le vivre ensemble à Sasaguri, une communauté de Kyūshū. Dans l'entrelacs des dynamiques du dedans et du dehors. pp. 1-25; doi : https://doi.org/10.3406/asie.2013.1414

https://www.persee.fr/doc/asie_0766-1177_2013_num_22_1_1414

Fichier pdf généré le 06/02/2019 


\title{
SASAguri. Dedans, DeHORS : \\ UNE APPROCHE ETHNOLOGIQUE COLLECTIVE
}

\author{
Anne Bouchy
}

Ce volume n'est pas une simple somme d'articles réunis autour d'une même thématique pour la publication. C'est le fruit d'une réflexion ethnologique partagée et appuyée sur une ethnographie collective, engagée par les auteurs en 2004 et réalisée en commun chaque année jusqu'en zoro dans la commune de Sasaguri 篠栗町 (département de Fukuoka 福岡県, dans le nord de Kyūshū 九州). Pour les membres de cette équipe d'ethnologues, ces contributions représentent donc aussi un travail en " retour » d'une partie de ce qu'ils ont appris en dialoguant avec les habitants de Sasaguri et en séjournant parmi eux au cours de toutes ces années. Et c'est au nom des membres de cette équipe que je brosse ici les grandes lignes de cette riche collaboration.

\section{La réalisation du programme de recherche}

Le projet de l'équipe franco-japonaise à l'origine de cette réalisation s'inscrit dans le prolongement d'autres collaborations antérieures, auxquelles avaient participé une partie de ses membres, depuis 1994, dans le cadre de programmes de recherche franco-japonais quadriennaux de l'Ecole française d'Extrême-Orient ${ }^{1}$. Le moteur décisif en a été la volonté exprimée par les ethnologues réunis lors de ces réalisations précédentes, de continuer à travailler ensemble, mais cette fois en s'appuyant sur une démarche ethnographique collective, autour d'une thématique fédérant leurs intérêts respectifs. C'est ainsi que se forma, dès 2000 , le noyau d'une nouvelle équipe constituée de chercheurs japonais et français, qui se mirent en quête à la fois de possibilités de travail de terrain au Japon et de thématiques fédératrices.

Parmi les objectifs majeurs qui furent nôtres dès le départ, il y avait le souhait de mettre en commun les méthodologies de l'enquête de terrain, les problématiques d'actualité au Japon et en France dans les champs de l'ethnologie du Japon, de l'anthropologie sociale et historique, et ce à partir de réalités locales qui feraient

I. Programme «Identités, marges, médiations », 1994-1999 (J.-P. Berthon, A. Bouchy, P. F. Souyri, éd., Identités, marges, médiations. Regards croisés sur la société japonaise. Actes des trois tables rondes franco-japonaises 1997-1998, coll. "Études thématiques » 10, Paris, EFEO, 2001, 318 p. ; et en japonais : A. Bouchy et Wakita Haruko 脇田晴子, éd., Aidentiti, shūen, baikai. Nihon shakai. Nichifutsu kyōdō purojekuto アイデンティティ、周縁、媒介・<日本社会〉甘仏共同プロジェクト, Tōkyō, Yoshikawa Kōbunkan 吉川弘文館, 2000, 282 p.). Programme "Légitimités, légitimations 》, 2000-2004 (A. Bouchy, G. Carré et F. Lachaud, éd., Légitimités, légitimations. La construction de l'autorité au Japon, coll. "Études thématiques » 16, Paris, EFEO, 2005, 317 p.). 
l'objet de nos investigations communes. D'emblée, une publication collective fut envisagée qui viendrait combler un manque, celui de l'approche ethnographique plurielle et franco-japonaise des réalités contemporaines d'une communauté locale, mais qui ne serait pas une simple monographie. Nous verrons comment nous avons tenté de résoudre cette équation.

Le choix de travailler à Sasaguri a été orienté par les effets contemporains d'un événement socioreligieux de la fin du XIX ${ }^{e}$ siècle : la formation d'une réplique du pèlerinage des 88 temples de Shikoku 四国 (comme il y en a un très grand nombre ailleurs dans le pays), qui fut ici le reflet d'un mouvement populaire local, et qui a eu pour effet une très dense concentration de tcmples ct d'oratoircs sur le tcrritoirc communal (plus d'une centaine), unique en son genre ${ }^{2}$. Bien que l'ensemble constitué par le pèlerinage et ses alentours ait été repéré par les ethnologues japonais depuis les années 1980 comme un exceptionnel «espace de pouvoirs subtils " (reinō cbitai 霊能地带) ${ }^{3}$, le second à l'échelle nationale après la région du mont Ikoma 生駒山 à l'est d'Ōsaka, jusque-là Sasaguri n'avait fait l'objet d'aucune recherche systématique de grande envergure. Plusieurs d'entre nous conduisant des recherches centrées sur le religieux, c'est cet aspect qui a d'abord attiré notre attention et été décisif dans le choix des lieux. Des investigations préliminaires nous ont vite convaincus que ce pèlerinage avait été à l'origine de multiples circuits de passage et d'échanges dans la communauté locale, et qu'il s'inscrivait en outre sur un arrière-plan local ancien, lui-même riche en éléments religieux, sociétaux, politiques et environnementaux, qui devaient faire partie intégrante de nos recherches. La mise en lumière des relations entre tous ces éléments pris dans des dynamiques de mobilités et de constructions identitaires à l'intérieur du contexte historique micro-local, régional et national, fut ainsi placée au centre de notre projet.

À la suite de consultations préliminaires qui s'étalèrent entre 2002 et 2003 , notre projet fut formulé et accepté en 2004 en tant que programme de recherche de l'EFEO pour les périodes 2004-2008, puis 2009-2012, sous le titre « Entre 'dehors' et 'dedans' : les dynamiques socioculturelles au Japon ». Durant la première enquête exploratoire qui eut lieu en octobre 2004, nous avons été guidés sur tout le parcours du pèlerinage de Sasaguri et dans les temples par les responsables religieux, et nous avons pu bénéficier d'une riche présentation historique, archéologique, environnementale et administrative de la commune et de son patrimoine culturel grâce aux responsables du Centre de documents historiques et de matériaux ethnographiques

2. Voir dans ce même volume la contribution de Nakayama Kazuhisa 中山和久, «La dynamique de création, réplication et déclin des lieux de pèlerinage : le nouveau pèlerinage de Shikoku à Sasaguri ", p. 29I-308.

3. Terme de Fujita Shōichi 滕田庄市, dans Shōzaki Ryōsei 庇崎良清, Omikuji. Shinbutsu no utsuwa to narite オミクジ——神仏の器となりて, Koshigaya 越谷 (Saitama-ken 埼玉紧), Kado sōbō かど創房, 2007 (éd. augmentée ; I re éd. 1993), p. 198. «[...] [Ce sont des] lieux où est concentré le plus grand nombre de spécialistes religieux actifs de toutes catégories, de pratiquants des rituels de toutes sortes 》 (Suzuki Masataka 鈴木正装, ci-après dans ce même volume, "Modernisation des temples bouddhiques et société locale : le Nanzō-in de Sasaguri », p. 416). 
de Sasaguri (Sasaguri chōritsu rekishi minzoku shiryō-shitsu 篠栗町浐歴史氏俗資料室). Dès mars 2005, commença l'enquête collective proprement dite.

Nous avions tous une longue pratique de l'approche ethnographique de la société japonaise, qu'elle soit individuelle ou en collaboration avec d'autres chercheurs, ou à visée pédagogique avec des étudiants. C'est pourquoi nous avons aussi cherché à cumuler les dimensions de la recherche et de la pédagogie, en faisant participer des étudiants dont chacun de nous dirigeait alors les travaux. Nous avons également souhaité rencontrer les ethnologues en poste dans les universités, ou autres institutions proches du lieu choisi, qui travaillaient sur des sujets et des terrains situés dans le cadre de la région. Plusieurs strates de l'équipe se sont ainsi formées, les membres permanents, les chercheurs invités, et les étudiants dont la participation a été plus ou moins longue.

Les fondements de cette entreprise collective et le ciment de l'équipe des membres permanents - Anne Bouchy, Ishikawa Toshiko 不川稔子, Kanda Yoriko 神田より子, Nakayama Kazuhisa 中山和久, Mori Hiroko 森弘子 et Suzuki Masataka 鈴木i正崇 — sont de plusieurs ordres. Tous, nous avons été formés en ethnologie du Japon dans des universités japonaises, au cours des années 1970, par ceux qui sont considérés comme des chefs de file dans cette discipline. Sous leur direction, nous avons participé à de nombreuses enquêtes de groupe dans toutes les régions du pays. Puis, sur ces modèles, réactualisés au fur et à mesure que se manifestaient dans les communautés locales et chez les individus les conséquences des transformations socioculturelles des années 1980, nous avons nous-mêmes organisé des travaux de terrain avec nos propres étudiants. Certains d'entre nous s'y sont d'ailleurs trouvés réunis. Nous partageons ainsi des connaissances et des pratiques que nous avons pu mettre à l'épreuve en de nombreuses occasions. Nous nous sommes tous également côtoyés dans diverses enquêtes ponctuelles portant sur de grands rites collcctifs au fin fond des montagnes ou au cœur des grandes villes, retrouvés dans des congrès, des colloques au Japon, en France et dans d'autres pays, invités mutuellement à participer à diverses activités pédagogiques. Nous avons lu réciproquement nos publications respectives. Avant de nous rendre ensemble à Sasaguri, nous nous connaissions donc bien et pouvions pressentir la façon dont chacun d'entre nous aborderait les thématiques communes. Aussi l'organisation du travail n'a-t-elle jamais posé de problème. Il faut bien sûr ajouter à ceci l'amitié qui nous lie et s'est renforcée au cours de toutes ces années durant lesquelles le travail à et sur Sasaguri nous a régulièrement réunis. Chacun a également apporté son expérience et ses compétences propres. Suzuki Masataka - dont l'approche en ethnologie du Japon est élargie d'une dimension d'anthropologie comparative par ses nombreux travaux sur les rites observés en Chine et en Inde - a réalisé des recherches majeures sur le fait religieux et notamment l'histoire des combinaisons associant les cultes des dieux et des bouddhas, sur le sbugendō 修験道 et les grands rites collectifs centrés sur les danses et le théâtre. Il lui revint de centraliser le travail dans le champ religieux, notamment pour le mont Wakasugi et le Nanzō-in, premier temple du pèlerinage de Sasaguri et paradigme extrême de la modernisation contemporaine des structures bouddhiques. Mori Hiroko nous a fait bénéficier de son expérience unique de l'ethnographie et de la micro-histoire de toute cette région, présentées 
notamment dans ses travaux sur le shugendō des monts Hiko 英彦山 et Hōman 宝満山 dont elle est la spécialiste, ainsi que de son insertion personnelle dans tous les milieux locaux du nord de Kyūshū, son lieu de vie, où elle fut pour nous une introductrice privilégiée. Elle assura en particulier la lecture et l'analyse de toutes les archives manuscrites concernant le shugendō de Wakasugi dont elle nous fit des comptes rendus réguliers. Nakayama Kazuhisa fut le maître d'œuvre des problématiques concernant le pèlerinage de Sasaguri, sur lequel il avait déjà travaillé au cours de ses travaux minutieux à l'échelle du Japon sur tous les pèlerinages, leurs réalités et leur approche théorique. Kanda Yoriko apporta un regard comparatif régional à partir de sa longue pratique ethnographique dans le nord-est du pays, où ses investigations l'ont conduite à traiter du fait religieux, du shugendō et des femmes spécialistes des oracles de cette région, et plus généralement de l'histoire des femmes, parallèlement à des recherches spécialisées dans les arts rituels et ludiques du spectacle et de la danse. Ishikawa Toshiko a une longue expérience des collectes et du traitement des diverses branches de la littérature orale, ainsi que des rites annuels, notamment dans la région du Japon central, et, par ce biais, elle s'est penchée sur la question des femmes dans la maison; c'est donc elle qui fut chargée de ces questions dans le cadre de la maison et des quartiers de Sasaguri. Anne Bouchy, qui assura la coordination du projet et de sa réalisation, centra son travail sur les différents types de relations coutumières au monde végétal. Par sa double formation ethnologique en France et au Japon, ainsi que par ses activités d'enseignement et de recherche dans ces deux pays, elle a contribué à développer le dialogue autour des manières japonaises et occidentales d'aborder les problématiques du fait religieux, du shugendō et des questions concernant l'environnement.

Dans le cadre de ce programme de recherche sur la commune de Sasaguri, six enquêtes de terrain annuelles de dix jours à plus de trois semaines chacune, financées en tant que parties constitutives du programme quadriennal de l'EFEO, furent menées par l'équipe entre mars 2005 et juillet 2010 . Nous avons également obtenu deux subventions de la Fondation du Japon (2005-2006 et 20II). Les six membres permanents furent rejoints par une étudiante française de l'université de Toulouse le Mirail à partir de 2009. Il fut également fait appel à des participants temporaires (six étudiants japonais des universités de Fukuoka, Kyūshū et Keiō, quatre étudiants français, deux chercheuses, l'une française et l'autre japonaise) soit durant nos séjours à Sasaguri, soit pour des enquêtes individuelles complémentaires durant les moments où nous ne pouvions être là pour observer des rites annuels ou faire le suivi régulier de pratiques coutumières. Plusieurs d'entre nous sont également venus pour des enquêtes individuelles répétées tout au long de ces années. Chacune de ces participations a donné lieu à des rapports annuels écrits, qui s'ajoutèrent aux innombrables fiches constituées par tous. À ceci se sont ajoutés sept séminaires de recherche (un à l'université Keiō, six à Sasaguri) auxquels furent conviés à intervenir de nombreux chercheurs travaillant sur les questions au centre de nos intérêts. Nous avons cherché également à élargir notre connaissance de la région en nous rcndant chaque année hors de Sasaguri, soit à des rites annuels avec des danses rituelles (comme le matsue 松会 de Hibaruyama 桧原山 [Usa 宇佐], le kagura 神楽 de Buzen 豊前 à Yama.uchi 山内 [Buzen], etc.), soit sur les lieux qui apparaissaient 
le plus fréquemment dans les propos de nos interlocuteurs et dans les documents. Enfin, un déplacement de l'équipe à laquelle se joignirent des membres du Centre d'anthropologie sociale de Toulouse (LISST-CAS) sur des sites de pèlerinage en Espagne (Núria, Montserrat), suivi d'une table ronde à l'université de Toulouse le Mirail (septembre 20II) plémentaire. Le travail d'écriture lancé en 2010-20Ir et poursuivi en 2012-2013, fut ensuite associé au travail de traduction et d'édition qui dura jusqu'en 2014 .

\section{Objets et objectifs}

L'opposition dedans/dehors est exprimée généralement par le couple de termes ucbi ウチ, le dedans, qui désigne aussi bien l'intérieur de quelque chose (uchi 中/内), que «ma/notre maison» (ucbi 家), «chez nous »et même « nous » ou « moi », et par là tout ce qui touche à l'intime; et sotoソト/外, ou yoso $コ$, 他所/余所, qui désigne le debors en tant qu'extériorité matérielle et psycho-sensible, les relations avec l'autre, et aussi l'altérité, la marginalité. Ces paires de contraires, le plus souvent données à penser comme irréconciliables, sont convoquées comme des éléments structurant les pratiques, les rites, les relations au monde. Elles ont servi à la construction des identités et aussi à celle de pratiques discriminatoires. L'instrumentalisation de l'antagonisme intériorité/extériorité se retrouve ainsi dans tous les registres de l'expérience et, par là, des observations faites sur le terrain. À ce titre, choisir un tel angle d'approche a été pour nous, tout d'abord, un moyen commode de fédérer nos démarches individuelles. Ceci nous a aussi permis de prendre nos distances avec le principe de la monographie locale, qui se focalise sur l'intérieur des réalités locales et cherche à donner un aperçu global de tous les aspects d'une communauté donnée. Pour cela, tout en travaillant à partir de nos enquêtes de terrain collectives portant sur l'ensemble des facettes de la vie locale, nous avons délimité un certain nombre d'axes plus spécifiquement explorés par chacun d'entre nous au cours des enquêtes successives et dans l'écriture. Selon les époques et les chercheurs, l'opposition dedans/dehors a été appréhendée de façons très diverses. Certains spécialistes de l'ethnologie du Japon, notamment, l'ont parfois réifiée ou prise pour une donnée de base plutôt que de la remettre en cause, alors que d'autres ont fait porter l'attention sur ses utilisations par des systèmes locaux. Aussi, dans un premier temps, chacun des auteurs a-t-il eu pour mission de mettre en lumière, sur le terrain et dans les archives, les faits d'antagonismes dedans/dehors et les dires concernant ceux-ci ; et également de questionner la façon dont les approches antérieures ont traité ces mêmes oppositions dans chaque thème étudié. Ceci nous a permis de voir la multiplicité et l'entrecroisement des cercles d'intériorité et d'extériorité dans lesquels se situent les individus et les groupes. Par là, nous avons été amenés à nous interroger sur ce qui détermine et fait fluctuer ce qui est donné comme la ou les frontière(s) du dedans

4. "Aux sources de la créativité : chemins et paysages des sites de pèlerinages » (I2 septembre 20II). 
et du dehors pour chacun d'eux et sur la façon dont ces frontières contribuent à construire les identités individuelles et collectives.

Ce choix s'est révélé efficace pour permettre à nos diverses approches d'échapper à une vision statique et simplement focalisée sur l'intérieur de la communauté locale de Sasaguri. Dans cette perspective, Nakayama Kuazuhisa explique bien ici la valeur heuristique d'une telle démarche et son intérêt dans un domaine comme les études sur le pèlerinage :

Cette analyse montre que, sur le phénomène du pèlerinage, opère fortement l'effet de transformation du dedans par le détournement de modèles du debors, c'est-à-dire que la signification du dehors joue un rôle crucial et déterminant. Jusqu'à présent, les travaux et les enquêtes de terrain en ethnologie du Japon se sont principalement et avant tout intéressés à l'état des choses à l'intérieur des communautés locales ; aussi les pratiques coutumières qui s'exportaient vers l'extérieur ont-elles eu du mal à accéder au rang d'objets d'étude. Or, comme les pratiques coutumières de l'extérieur, où sont à l'œuvre des dynamiques différentes de celles qui existent dans les pratiques internes, possèdent elles aussi un sens majeur, il faudrait qu'à l'avenir, elles soient largement prises en compte par les recherches.

L'ethnologie du Japon, dès ses débuts, a pris en compte la question de l'intériorité et de l'extériorité. Mais si, par là, les processus ayant présidé aux phénomènes d'exclusion ont ainsi été mis en évidence, l'attention a également pu être attirée sur les liens établis entre des pôles « construits comme » exclusifs. Les groupes sociaux et le religieux figurent parmi les grands axes adoptés par les travaux sur la question. Pour n'évoquer que quelques approches déterminantes pour la suite des recherches, Yanagita Kunio 柳田国男 s'est intéressé dès ses premières études aux groupes isolés et aux populations périphériques de l'archipel, gens des montagnes, itinérants, et plus tard gens de mer, qui jusque-là n'avaient pas été étudiés ${ }^{6}$. Il a ouvert par là des pistes pour une approche cherchant à éclairer l'interdépendance historique, économique et culturelle de ces groupes et des autres éléments de la population, notamment les agriculteurs sédentaires se considérant comme les Japonais « du dedans " par opposition à ceux-ci. À la même époque, Orikuchi Shinobu 折口信夫 démontrait la valorisation non seulement de l'ailleurs, du dehors et de l'étranger, mais aussi des mobilités et des échanges entre le dedans et le dehors, en théorisant la notion de (dieux/ancêtres) "visiteurs ", à partir des ritualités des îles du sud du Japon et notamment d'Okinawa ${ }^{7}$. Mais Yanagita, pour tenter de contribuer à la

5. Dans ce même volume, Nakayama Kazuhisa, « La dynamique de création, réplication et déclin des lieux de pèlerinage : le nouveau pèlerinage de Shikoku à Sasaguri ", p. 323.

6. Yanagita Kunio 柳[H]国]男, Nocbi no karikotoba no ki 後狩詞乱, 1909, P. I-39; Kijiya monogatari 木地坛物語, 1911, dans Teibon Yanagita Kunio shü 定本柳田国男集, vol. 27, Tōkyō, Chikuma

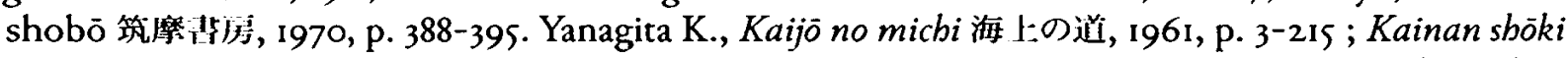
游南小部, 1921, p. 223-379; Shima no jinsei 结の人生, 196I, dans Teibon Yanagita Kunio sbū, vol. I, Tōkyō, Chikuma shobō, 1968, p. 387-498. Sur Yanagita et la formation de l'ethnologie du Japon, voir aussi A. Bouchy, "De l'ethnologie du Japon : par qui, où, comment ?", Ateliers 30, Nanterre, Laboratoire d'ethnologie et de sociologie comparative, université de Paris X, 2006, p. 63-99.

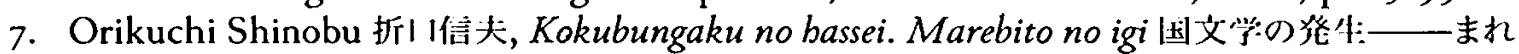
びとの意義, 1929, dans Orikuchi Shinobu zenshū 折门信夫全集, vol. 1, Tōkyō, Chūō kōron-sha 中以 公論社, 1993, p. 3-62. 
reconstruction sociale et humaine après la longue période des guerres du $\mathrm{Xx}^{\mathrm{e}}$ siècle, a ensuite orienté ses travaux vers les communautés villageoises et la maison-famille. C'est ainsi que, s'inscrivant dans ce prolongement, la ligne majeure de l'ethnologie du Japon s'est focalisée sur ce «dedans » central de la société, ancré dans les valeurs de la sédentarité et de la riziculture.

Néanmoins, des pionniers comme Tsuboi Hirofumi 坪井洋文 ${ }^{8}$ et Sasaki Kōmei 佐々木高', en engageant des recherches sur les montagnards pratiquant les cultures sur brûlis, ont développé les premières intuitions de Yanagita et ouvert des pistes de réflexion extrêmement riches sur l'hétérogénéité sociale. Ils ont contribué ainsi à remettre en cause l'idée prégnante d'une société japonaise homogène. Le médiéviste Amino Yoshihiko 網野善彦 a, lui aussi, largement contribué à la création de divers courants de recherche en histoire et en ethnologie sur l'« autre », le "différent » (ijin 異人) ou l'exclu, et sur les différentes façons dont celui-ci est de fait imbriqué dans la sociétée ${ }^{10}$. À leur suite, de nombreux auteurs se sont penchés sur les réalités des populations marginalisées, discriminées ou des itinérants. Parmi eux, on peut citer Oguma Eiji 小熊英”, spécialiste de sociologie historique, qui examine la généalogie de l'idée de Nation homogène et sa construction sur arrière-plan du nationalisme de l'après-guerre ${ }^{11}$. Le champ de ces études est ainsi largement ouvert aujourd'hui, et s'étend sur toute la palette des rapports sociaux et économiques, du politique, du religieux, de l'oralité, et bien d'autres. L'objectif du présent travail se situe, quant à lui, à une échelle restreinte, celle d'une communauté locale, de sa construction en tant que telle par rapport aux autres localités et entités extra-locales, et par rapport aux divers groupes qui la constituent, ainsi que celle de l'espace-temps local dans lequel se déploie une multitude d'échanges et de relations.

Comme l'indique le titre du volume, Le vivre ensemble à Sasaguri, une commune de Kyüshü. Dans l'entrelacs des dynamiques du deduns et du debors, ce que nous avons surtout cherché à comprendre, c'est ce qui se passe entre les multiples «dedans » et « dehors », et ce, dans tous les sens. Par-delà les oppositions statiques et dualistes, ce sont donc les dynamiques relationnelles qui sont au cœur de notre travail : ces maillages tissés par toutes sortes d'extériorités et d'intériorités enchevêtrées qui interagissent les unes avec les autres. Prendre en compte les limites et les frontières, les espaces et les entités a ici pour corollaire essentiel la mise en lumière des relations qui composent, lient, divisent ces ensembles, ainsi que les mouvements,

8. Tsuboi Hirofumi 坪井洋文, Imo to Nibonjin イモと日本人, Tōkyō, Miraisha 未米社, 1979 ; Tsuboi, Ine wo eranda Nibonjin 稻を選んだU本人, Tōkyō, Miraisha, 1982.

9. Sasaki Kōmei 仿々木商明, Inasaku izen 稲作以前, Tōkyō, NHK Books, 197ı ; Sasaki, Shōyōjurin bunka no michi 然葉樹林文化の道, Tōkyō, NHK Books, 1982.

IO. Amino Yoshihiko 網野善彦, entre autres travaux, Muen, kugai, raku. Nibon chüsei no jiyū to beiwa 無縁·公界·楽——日本中世の白由と平和, Tōkyō, Heibonsha 平凡社, 1978 (réédition augmentée 1987); Amino, Nibonron no shiza. Rettō no shakai to kokka 日本論の視座——列鼠の社 会と国家, Tōkyō, Shōgakkan 小学館, 1990 (réédition 2000).

II. Oguma Eiji 小熊英-, notamment dans Tan.itsu minzoku shinwa no kigen. "Nibonjin » no jigazō no keifu 单一比族神話の起源——山本人>の自画像の系譜, Tōkyō, Shin.yōsha 新矅社, 1995; "Nihonjin » no kyōkai. Okinawa, Ainu, Taiwan, Chösen. Shokuminchi sbibai kara fukki undō made

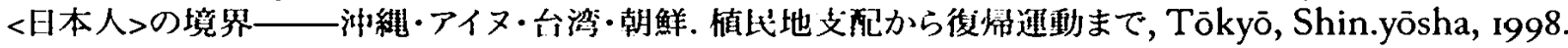


les passages et les dynamiques qui les traversent. Les deux notions dedans/dehors sont donc appréhendées ici comme des catégories complémentaires, non exclusives et inséparables. En ceci notre position rejoint celle que Jean-Pierre Vernant adopte dans son analyse du couple Hestia-Hermès dans le contexte de la Grèce ancienne ${ }^{12}$.

En outre, nous avons aussi tenté de faire porter notre attention sur les emboîtements de différents types d'intériorité et d'extériorité cumulés par un même sujet individuel ou collectif, afin de voir comment celles-ci s'articulent les unes avec les autres, pour un même sujet ou entité ; ou encore comment, en différents contextes, s'organisent les positions respectives de ces entités à multiples niveaux d'intériorité et d'extériorité. L'importance de la question des relations qui forment le maillage complexe de la vie sociale a été mise en avant par des travaux comme ceux de Gregory Bateson ou de Philippe Descola ${ }^{13}$. Néanmoins, plutôt qu'une étude concernant les schèmes de l'expérience, plus modestement, notre travail s'est donné comme objectif la recherche, la plus précise possible, des processus concrets déployés dans la vie coutumière de Sasaguri, pour élaborer, reconstruire, transformer les formes socioculturelles de ces multiples dedans/dehors. Ceux-ci ont été abordés à différentes échelles plus ou moins imbriquées les unes dans les autres : l'intimité individuelle, l'individu et la maison, les divers groupes locaux, les entités villageoises, la commune et les communes limitrophes, la région, le pays, l'Asie orientale et même l'échelle internationale avec l'histoire moderne et l'actualité contemporaine. Nous avons cherché à comprendre quels sont aujourd'hui, et ont été au fil du temps et des ruptures historiques, les impacts réciproques des dynamiques issues des divers groupes sociaux en présence sur ces groupes eux-mêmes et sur les autres.

Par-delà l'opposition dedans/dehors, c'est l'émission croisée des forces (de changement, continuité, rupture, recomposition), leur réception et leurs effets aux divers niveaux d'intériorité et d'extériorité qui sont donc au cœur de notre questionnement. Dans cette optique, a été très stimulante la remise en cause des mécanismes de construction des « communautés " par Tim Ingold. Regardant plutôt celles-ci comme l'entrecroisement de multiples trajectoires individuelles, ce dernier insiste en effet sur le déploiement des lignes de mobilité et des osmoses. Allant même jusqu'à nier l'existence d'un dedans et d'un dehors, il prône la construction d'une « écologie de la vie ", dont l'objet doit être de s'enquérir « not of the relations between organisms and their external environments but of the relations along their severally enmeshed ways of life ${ }^{14}$ ». En intégrant la dimension historique et en resituant Sasaguri dans les divers contextes locaux, nationaux et internationaux, nous avons essayé de suivre, dans son déroulement vivant, le fil des dynamiques qui ont

I2. Jean-Pierre Vernant, "Hestia-Hermès. Sur l'expression religieuse de l'espace et du mouvement chez les Grecs ", L'Homme, tome 3, n 3, 1963, p. 12-50. En reprenant partiellement le titre du numéro d'une revue qui lui est consacré (Le genre bumain, $n^{\circ} 53$ - "Jean-Pierre Vernant. Dedans dehors ", Is février 2013), cette présentation se voudrait à la fois un hommage à l'anthropologue de la Grèce ancienne, un rappel de la thématique du programme de recherche et l'expression de la position de notre équipe parmi les habitants de Sasaguri.

13. Philippe Descola, Par-delà nature et culture, Paris, Gallimard, 2005, p. 137-144.

14. Tim Ingold, Lines: A Brief History, Londres / New York, Routledge, 2008, p. I03. 
présidé à l'entrecroisement des constructions et recompositions locales, lesquelles ont elles-mêmes produit des effets en retour.

\section{Appréhender la commune de Sasaguri 篠栗町}

Dès que l'on interroge les réalités micro-locales dans leurs rapports à la société (intra-locale, régionale, nationale), aux pouvoirs, aux grands événements, se dessinent évidemment les dimensions du «dedans » et du « dehors »; néanmoins celles-ci ne sont nullement simples. Multiples, et même se démultipliant, elles sont présentes et s'entremêlent à toutes les échelles de l'analyse, depuis les dimensions territoriales, administratives, politiques, jusqu'à celles de la famille, de l'individu et de toutes les constructions socioculturelles.

Pour en comprendre les dynamiques relationnelles, nous sommes partis en premier lieu de deux niveaux d'intériorité et d'extériorité. Le premier est celui qui est défini par les délimitations territoriales et administratives qui tracent des démarcations entre les entités locales tout en les réunissant dans un même espace communal ; le second est celui des groupes indépendants des statuts administratifs.

\section{Délimitations territoriales et administratives}

La commune de Sasaguri ${ }^{15}$ est située au nord-ouest du département de Fukuoka ${ }^{16}$, dans le canton de Kasuya 糟屋郡 (cartes I et 2). Elle se trouve à $12 \mathrm{~km}$ de Fukuoka, sixième ville du pays, qui fut dès l'Antiquité la porte principale des échanges avec le continent asiatique, et à laquelle elle est reliée par une ligne ferroviaire directe. La superficie de la commune est de $38,90 \mathrm{~km}^{2}$ (389o ha) (E-O : $\left.8 \mathrm{~km}, \mathrm{~N}-\mathrm{S}: 7 \mathrm{~km}\right)$, dont plus de $60 \%$ sont occupés par des espaces montagneux couverts de forêts. Sa population actuelle est de 31 532 habitants (2012), qui habitent pour la plupart dans la partie urbanisée à l'ouest de la commune, près de la gare de Sasaguri. Beaucoup d'entre eux vont travailler quotidiennement à Fukuoka, ce qui vaut à Sasaguri la qualification de «bed-town » de la mégalopole. Le secteur tertiaire des activités de service est en pleine expansion comme ailleurs, et représente aujourd'hui presque $80 \%$ des activités, tandis que le secteur primaire (agriculture, sylviculture) a diminué jusqu'à être réduit à $1,2 \%$ et le secteur secondaire à un peu plus de $20 \%$. La force d'attraction du pèlerinage du nouveau Shikoku, qui jusque dans les années 1990 drainait environ un million de visiteurs par $\mathbf{a n}^{17}$, est en baisse depuis une décennie, ce qui a forcé la commune à se tourner vers une nouvelle exploitation de ses ressources forestières matérielles (usage des bois de détourage) et sociales (organisation d'espaces récréatifs, de chemins de randonnées et de « thérapie forestière »).

15. $130^{\circ} 31^{\prime}$ de longitude est, et $33^{\circ} 37^{\prime}$ de latitude nord.

16. $4976,97 \mathrm{~km}^{2}$, pour plus de 5 millions d'habitants.

17. Évaluation locale. Voir dans ce même volume, Nakayama Kazuhisa, « La dynamique de création, réplication et déclin des lieux de pèlerinage : le nouveau pèlerinage de Shikoku à Sasaguri ", p. 284-287. 


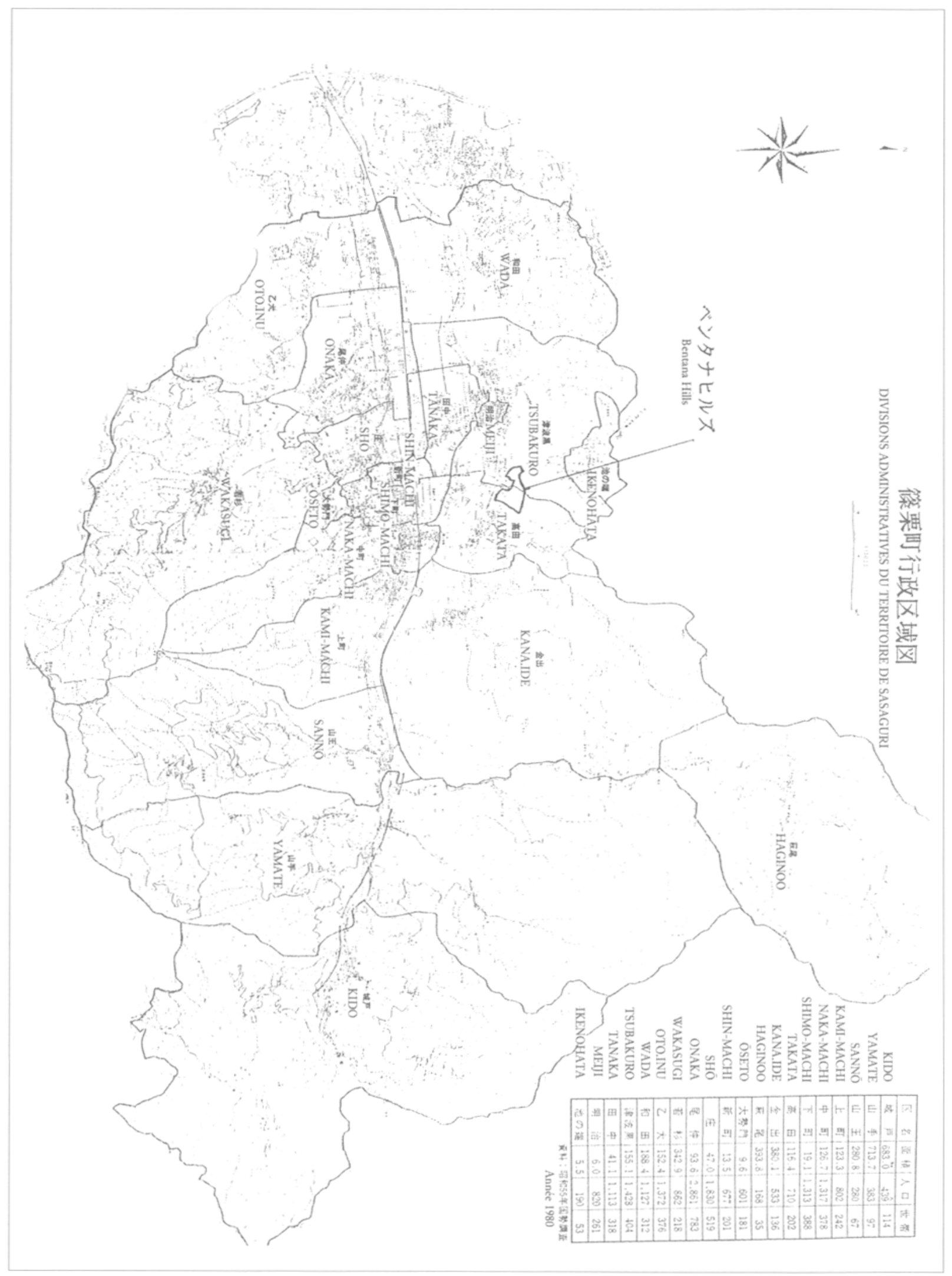

Carte 1 : Circonscriptions administratives de Sasaguri. 
La commune est traversée d'est en ouest par la rivière Tatara 多々良川. La partie occidentale, qui bénéficie de l'eau des montagnes se dressant au sud, est une petite cuvette où s'étendent à la fois les espaces agricoles (rizières irriguées et champs) et le centre urbain de la municipalité, les uns et les autres résultant d'une installation très ancienne attestée par les fouilles archéologiques. Seule l'extrémité de ce côté ouest est limitrophe de territoires de plus en plus urbanisés (arrondissement Higashi de Fukuoka 福岡市東区, commune de Kasuya 粕屋町). Mais tout le reste du pourtour communal, qui est fait de collines et de montagnes, jouxte des espaces majoritairement constitués de montagnes boisées des communes avoisinantes (Hisayama 久山, Wakamiya 若宮, Iizuka 飯塚, Sue 須恵).

Il y a donc un fort contraste résultant de la coexistence sur un même territoire, d'une part, de cette urbanisation ancienne - mais fortement concentrée dans un espace limité - et d'autre part, de l'extension des zones montagneuses non habitées ou à habitat dispersé et peu dense. Topographiquement, le territoire communal paraît clos sur lui-même par les espaces montagneux de ses côtés nord, est et sud. Mais il est en réalité lié depuis une date ancienne à toute la région environnante par plusieurs voies de circulation (carte 2). La commune est traversée d'est en ouest par la nationale 20r et la départementale 607, ainsi que par la ligne de chemin de fer de Sasaguri. La route fut ouverte dès le début du XVII ${ }^{e}$ siècle pour permettre les allées et venues entre l'ouest et l'est du fief de Fukuoka (ou de Chikuzen 筑前), dit aussi fief des Kuroda 黒田藩, du nom des daimyō 大名 qui le gouvernèrent tout au long d'Edo (de 1600 à I871). Appelée Route de Sasaguri, Sasaguri kaidō 策栗 街道, elle reliait directement la ville-sous-le-château de Fukuoka à la grande voie intérieure nord-sud de Kyūshū qui allait vers Honshū. Par sa position médiane sur le Sasaguri kaido, Sasaguri devint une localité-étape (shukuba macbi 宿場町), avec des structures d'hébergement et d'accueil des voyagcurs. La ligne ferroviaire reliant Sasaguri à Fukuoka a été ouverte en 1908 au moment de l'essor des mines de charbon de la commune. Puis, en 1968, le tunnel percé sous la montagne en a permis la jonction avec les voies ferrées de la partie orientale de cette région reliant le nord et le sud de Kyūshū. La départementale 92 assure le passage vers le nord. En outre, les montagnes ont été des espaces de circulation tant que la marche a été le moyen de déplacement ordinaire ${ }^{18}$. C'est aussi là que s'est déployé le nouveau pèlerinage de Shikoku à Sasaguri ${ }^{19}$ et qu'aujourd'hui se développe le tourisme vert des citadins, notamment sous une nouvelle forme, la « thérapie forestière ${ }^{20}$ ».

Administrativement parlant, Sasaguri est " une communauté " récente. Aujourd'hui la commune est composée de vingt-et-un quartiers $(k u 区)^{21}:$ Kana.ide 金出, Takata

I8. Au sujet de la circulation et des communautés " par-delà les montagnes ", voir dans ce même volume Anne Bouchy, «Les rapports communautaires aux espaces forestiers entre politiques du dehors et stratégies du dedans : les montagnes-forêts de Sasaguri ", p. 119-r29.

19. Voir dans ce même volume Nakayama Kazuhisa, «La dynamique de création, réplication et déclin des lieux de pèlerinage », p. 280-284.

20. Voir dans ce même volume Anne Bouchy, op. cit., p. 188-193.

21. Dans une ville, ce terme correspond à un arrondissement, notion qui s'accorde mal avec les réalités villageoises. La traduction de $k u$ par " quartier » reflète mieux l'individualité et la 


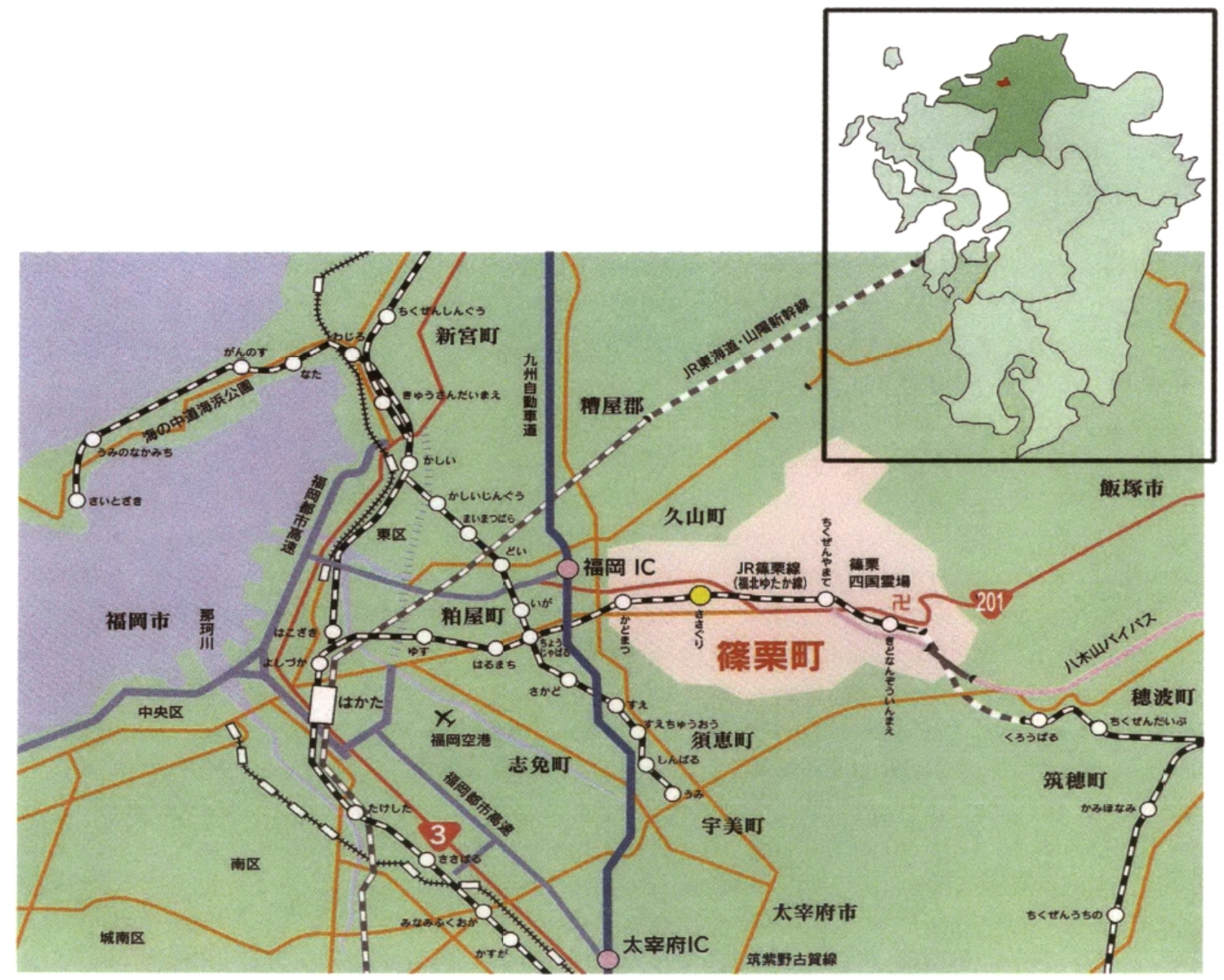

Carte 2 : Voies de circulation actuelles. (Ne sont indiqués que les principaux toponymes et ceux qui apparaissent dans les textes du volume.)

$\begin{array}{llll}\text { 篠栗町 } & \text { Sasaguri } & \text { 香椎 } & \text { Kashii } \\ \text { 久山町 } & \text { Hisayama } & \text { 福岡市 } & \text { Fukuoka } \\ \text { 新宮町 } & \text { Shingū } & \text { 東区 } & \text { Arrondissement Higashi de Fukuoka } \\ \text { 筑穂町 } & \text { Chikuho } & \text { 南区 } & \text { Arrondissement Minami de Fukuoka } \\ \text { 粕屋町 } & \text { Kasuya } & \text { 福岡空港 } & \text { Aéroport de Fukuoka } \\ \text { 粕屋郡 } & \text { Canton de Kasuya } & \text { 博多 } & \text { Gare de Hakata } \\ \text { 筑前山手 } & \text { Chikuzen-Yamate } & & \\ \text { 飯塚市 } & \text { Iizuka } & \text { 篠栗線 } & \text { Ligne de Sasaguri } \\ \text { 志免町 } & \text { Shime } & \text { 城户南蔵院前 Kido-Nanzō-in-mae } \\ \text { 太辛府市 } & \text { Dazaifu } & \text { 国道201号 } & \text { Nationale 2oI } \\ \text { 須恵町 } & \text { Sue } & \text { 九州自動車道 Autoroute de Kyūshū }\end{array}$


高田, Haginoo 萩尾, Onaka 尾仲, Wakasugi 若杉, Oto.inu 乙犬, Wada 和田, Tsubakuro 津波黒, Tanaka 田中, Sannō 山王, Kido 城戸, Yamate 山手, Shō 庄, Shin-machi 新町, Ōseto 大勢門, Kami-machi 上町, Naka-machi 中町, Shimo-machi 下町, Meiji 明治, Ike-no-hata 池の端, Bentana hiruzu ベンタナヒルズ22. Les dix premiers sont des anciens villages qui ont été réunis au cours de l'histoire moderne dans le mouvement de regroupement administratif et de fusion des communes. Les autres ont été créés par sectionnement d'une partie des anciens quartiers, au début puis au cours du $\mathrm{XX}^{\epsilon}$ siècle, avec l'augmentation de la population. Jusqu'en $\mathrm{I} 889$ (Meiji 22), les anciens villages de l'époque d'Edo (Sasaguri, Kana.ide, Takata, Haginoo, Onaka, Wakasugi, Oto.inu, Wada, Tsubakuro, Tanaka) conservent leur statut sous l'appellation de "petites circonscriptions » regroupées en plusieurs grandes circonscriptions, dont l'organisation a été remaniée trois fois entre 1873 et 1876 , pour finalement retrouver en 1885 leur nom de villages (mura 村) englobés dans le canton de Kasuya. Puis trois vagues de fusions communales à l'échelle du pays sous impulsion gouvernementale ont totalement restructuré le paysage administratif local ${ }^{23}$. Dans le cadre de la première, en I889, les quatre villages de Sasaguri, Kana.ide, Takata et Haginoo se regroupent pour former le village de Sasaguri 策栗村 (2448 habitants), et les six villages d'Onaka, Wakasugi, Oto.inu, Wada, Tsubakuro et Tanaka, celui de Seto 势門村 (2345 habitants). La logique de ces deux regroupements repose sur l'histoire liant mutuellement ces villages qui se jouxtent géographiquement. À la suite du remaniement des municipalités de 1927 , le village de Sasaguri prend le statut de commune, Sasaguri-chō 篗栗町. Enfin, en 1955, naît la municipalité actuelle de Sasaguri par fusion administrative de la commune de Sasaguri et du village de Seto. Mais Sasaguri a renoncé à participer à la quatrième étape des regroupements communaux (lancée en 2000), qui l'aurait contraint à fusionner avec plusieurs municipalitćs environnantes.

cohérence (sociale, historique, économique, culturelle) de chacun d'eux par rapport aux autres, et le fait que chaque quartier est aussi un élément d'un tout qu'est la commune de Sasaguri. À ce titre, " quartier » nous a paru meilleur que " circonscription ", dont la connotation est principalement administrative. "Section » désigne les divisions internes au quartier. Sur le détail des composantes des quartiers, voir dans ce même volume la contribution d'Ishikawa Toshiko, "Les rites annuels de la maison et des communautés locales en transformation : intérieur et extérieur des lieux de vie communautaires ", p. 497.

22. Population en 2013 : Kido 287 habitants, Yamate 216, Sannō 224, Kami-machi 715, Nakamachi 1786 , Shimo-machi 1622 , Takata 862, Kana.ide 475, Haginoo 137 , Ōseto 512 , Shin-machi 1 463, Shō 4310, Onaka 4712, Wakasugi 941, Oto.inu 3084 , Wada 3122, Tsubakuro 2 231, Tanaka 2075, Meiji 490, Ike-no-hata 231, Bentana hiruzu 2 I10 (mairie de Sasaguri). (Population totale en $1898: 4993$ habitants; en 1935: 10612 habitants; en 1960:14039 habitants).

23. Cette politique a réduit considérablement le nombre des entités administratives territoriales de tout le Japon, qui passent de 71 314 en 1888, à 15859 en 1889, puis à 10 520 en 1945, et 4668 en 1956. À l'issue de la dernière grande campagne de fusion de Heisei, elles ne sont plus que 1719 en 2014 (site du ministèrc des $\Lambda$ ffaires internes et des Communications : <http://www.soumu.go.jp/ gapei/gapei2.html>, dernier accès le 5 mars 2014). Sur l'impact local des fusions communales, voir dans ce même volume Anne Bouchy, "Les rapports communautaires aux espaces forestiers entre politiques du dehors et stratégies du dedans : les montagnes-forêts de Sasaguri », p. 162-165. 
Shō est dès avant 1889 une sous-division de Seto et le restera. Shin-machi s'est séparé de Shō en 1943 (sous le nom de Marubayashi-ku 丸林区) et prend son nom actuel lors de la création de la commune en 1955. Ōseto, où se développe une houillère dès les années 1910, se sépare en 1955 de Naka-machi, en tant que quartier de Sasaguri. Kami-machi et Shimo-machi se trouvent dans le périmètre du village de Sasaguri, ancienne étape de la route de Sasaguri, où à l'époque d'Edo il était interdit de construire des maisons particulières. Ces deux quartiers ont été créés fin Meiji, dans le contexte de l'urbanisation croissante, après l'ouverture de la ligne ferroviaire, puis, pour les mêmes raisons, en 1931 une partie de leur territoire devint le quartier de Shimo-machi. Meiji et Ike-no-hata sont deux quartiers dont l'histoire est liée à celle des mines de Sasaguri ${ }^{24}$, mais ils n'existent comme quartiers administratifs que depuis $196 \mathrm{r}$, l'année qui suivit la fermeture définitive de la mine Meiji-Takata 明治高田炭坑. Sannō, Kido et Yamate ont toujours fait partie du village de Sasaguri et sont devenus nominativement des quartiers dans les restructurations du XXe siècle. En 1956, Kido fut agrandi à son extrémité sud-est d'une partie de Naijū 内住, antérieurement intégrée à la commune voisine de Chikuhō 筑豊. Enfin, Bentana hiruzu ベンタナヒルズ, quartier résidentiel constitué de grands immeubles, vit le jour en 1996.

L'historique de ces quartiers montre ainsi que l'entité communale actuelle est multi-composée, et constituée en réalité d'un emboîtement de dedans et de dehors, tous relatifs à leurs positions et statuts respectifs, et dont les dynamiques complexes doivent impérativement être prises en compte lorsqu'on les étudie. Dans un tel contexte se pose la question du sens de la communauté à l'intérieur de la commune actuelle de Sasaguri, et de ce que recouvrent les notions d'intérieur et d'extérieur. Alors qu'en réalité Sasaguri n'est autre que la réunion de petites entités locales distinctes, on peut sans doute convenir avec Benedict Anderson que l'histoire moderne a construit la « communauté imaginée » dont cette commune revendique l'identité aujourd'hui dans son discours officiel tant face à l'extérieur qu'auprès de ses propres habitants ${ }^{25}$. En réalité, en tant qu'anciens villages, ces quartiers représentent bien plus que de simples unités administratives. Ce sont des communautés socioculturelles à identité micro-locale forte, dont, pour certaines, les modes de vie ont été jusqu'à aujourd'hui très contrastés. Ainsi, pour les citadins du centre de Sasaguri, Haginoo, ancien village de forestiers, posséderait une cohésion et un esprit que l'on ne retrouve plus guère ailleurs, alors même que ses propres habitants trouvent que ces caractères locaux sont précisément en train de disparaître du fait de l'intégration progressive des modes de vie urbains.

24. Sur l'exploitation minière à Sasaguri, voir la contribution de Kanda Yoriko 神田より \}, «Femmes de Sasaguri : au fond du religieux et à l'ubac du séculier ", p. 585-595.

25. Benedict Anderson, Imagined Communities: Reflections on the Origin and Spread of Nationalism, Londres, Verso, 1991, édition révisée et augmentée; édition française : L'imaginaire national. Réflexions sur l'origine et l'essor du nationalisme, trad. Pierre-Emmanuel Dauzat, Paris, La Découverte, 1996. 


\section{Autres types d'espaces et de groupes}

Au second niveau des intériorités et extériorités, on rencontre tous les autres types de groupes et de communautés déterminés par rapport à un centre et aux réseaux qui lient leurs membres entre eux. Ils sont nombreux. On évoquera ici seulement quelques-unes des pistes que nous avons suivies.

Il existe des groupes dont les limites internes et externes sont clairement définies. Ce sont les paroissiens (danka 檀家) de chacun des temples paroissiaux, le groupe des ujiko 氏子 soutenant un même sanctuaire tutélaire local, les multiples associations, les familles des morts d'un village qui utilisent encore un cimetière ou un columbarium communautaires, les usagers de communaux forestiers, et même le groupe de ceux qui pratiquent l'ascèse de la cascade dans un lieu particulier, par rapport à tous ceux qui sont extérieurs à cette pratique, etc.

Mais se rencontrent aussi des communautés translocales à cheval sur différentes limites internes/externes : c'est le cas des groupes fidèles des spécialistes religieux charismatiques, des groupes de pèlerins réunis autour d'un moine de l'une des 88 étapes du pèlerinage de Sasaguri ou autour d'un leader charismatique de l'extérieur, des pratiquants shugen 修験 qui traversent les espaces montagneux de toute la région et unissent ceux-ci ainsi que les villages situés le long de leur parcours en une totalité rituellement et socialement signifiante, des groupes familiaux liés de longue date à des villages extérieurs à Sasaguri par le biais des pratiques matrimoniales traditionnelles, ou encore des familles des soldats morts à la seconde guerre mondiale qui ont des réunions annuelles, sur place et hors de la commune, avec des groupes de l'extérieur de Sasaguri.

À l'inverse, il existe également des relations internes/externes reposant sur la multi-localisation d'individus ne formant pas communauté (dans le sens où les membres n'ont pas nécessairement de liens entre eux), mais qui ont un point d'ancrage commun à Sasaguri (et donc extérieur, pour eux, mais aussi intérieur, pour les gens de Sasaguri), comme par exemple les acheteurs de niches dans les columbariums des temples de pèlerinage (le Nanzō-in 南蔵院, entre autres), ou tous ceux (dispersés à l'échelle du pays) qui ont acheté des concessions dans les nouveaux cimetières collectifs installés dans la commune par des entreprises extérieures.

La question n'est que peu abordée dans le volume, mais nous nous sommes aussi interrogés sur la nouvelle redéfinition du rapport intérieur/extérieur qu'engendrait l'impact d'internet et des NTIC, efficaces et rapides instruments de rassemblement, de diffusion mais aussi de démantèlement du sens des communautés restreintes (localisées) et élargies (délocalisées).

En tout cas, là aussi, la multiplicité est la règle. La définition de l'intériorité et de l'extériorité change selon la position du sujet par rapport à Sasaguri, sur le plan de la résidence, selon les modalités de sa participation à la vie sociale, religieuse, etc. Un même individu peut occuper différentes positions d'intériorité et d'extériorité, et doit parfois gérer les antagonismes dus aux diverses appartenances. Il peut se situer dans la communauté d'un quartier, mais à l'intérieur de celui-ci être extérieur à divers groupes (religieux, familiaux, sociaux) internes au même quartier, ou être géographiquement extérieur à Sasaguri par son habitat mais, à l'inverse, être 
impliqué au plus au point dans un groupe de Sasaguri, de fidèles ou disciples d'un même spécialiste religieux charismatique, par exemple.

C'est donc aux façons d'être au milieu de ces divers groupes locaux, communautés, associations que nous nous sommes intéressés, notamment par le biais des fluctuations ou de la stabilité des appartenances. Il semble ainsi qu'aujourd'hui le partage d'un sentiment d'appartenance au groupe social du quartier ne soit plus toujours évident. Cela se manifeste, par exemple, lors de rites annuels comme celui de la fête des morts en été, obon 拈盆 (rebaptisée depuis quelques années "fête de l'été ", natsumatsuri 夏祭り). Traditionnellement, c'est l'un des moments les plus forts de l'expression rituelle du vivre ensemble de la communauté locale. J'ai en mémoire deux images contrastées de la danse du soir du bon au mois d'août 2010. À Haginoo ou à Kana.ide, quartiers où prévaut encore une forte solidarité locale, toute la population du centre du quartier était rassemblée en un même cercle pour des danses où se mêlaient enfants, jeunes, adultes et anciens, devant l'autel collectif où avaient été disposées les photos des morts de l'année. Ce cercle de danse, qui joint les vivants dans une même mémoire des morts qu'ils reconduisent ainsi dans l'au-delà, est à l'image de la cohésion du groupe. Mais dans l'un des quartiers urbanisés du centre de la commune, où se sont implantés de nombreux immeubles dont les résidents sont en majeure partie venus de l'extérieur, il a été totalement impossible aux participants de former un cercle de danse. Il y a eu toutes sortes de performances, des petits groupes dansaient plus ou moins ensemble, mais la sono bruyante n'a pas pu les rassembler en une danse commune. La question est alors de savoir dans quel sens et comment se déplacent les liens sociétaux.

\section{Les mouvements et les mobilités}

La troisième dimension qui nous a retenus est celle des mouvements qui traversent tous ces ensembles. Ces mobilités peuvent être observées sous l'angle du support matériel, comme par exemple le réseau de voies de circulation évoqué précédemment, par lequel transitent personnes, biens et informations. Le mouvement est ici de plusieurs sortes : de l'extérieur vers l'extérieur de Sasaguri pour tous ceux qui ne font qu'emprunter les routes et la ligne ferroviaire, à toutes époques; de l'intérieur vers l'extérieur, avec un retour vers l'intérieur pour les gens de Sasaguri (hier : les commerçants ambulants, les paysans allant vendre leurs productions à Hakata 博多 et en rapporter les eaux de vidange utilisées comme engrais, etc. ; aujourd'hui : les employés, les écoliers, etc.) ; de l'extérieur vers l'intérieur, avec un retour vers l'extérieur pour tous les pèlerins, les commerçants ambulants, les démarcheurs, les touristes, les citadins en quête de verdure, etc. Enfin il peut s'agir de passages restreints ou conduisant à arpenter une plus ou moins grande partie du territoire communal (une partie seulement, quelques-unes ou la totalité des 88 étapes du pèlerinage). Et ces passages peuvent être topographiquement et temporellement différents : ponctuels (au centre ou ailleurs), sur une partie plus ou moins étendue, ou sur les seuls bords de la commune (le parcours rituel des yamabushi 山伏); réguliers, épisodiques, aléatoires, uniques, etc. 
Mais nous avons aussi envisagé ces mouvements transversaux entre les groupes sous l'aspect des dynamiques que produisent soit des changements dans les conditions internes à tous les groupes précédemment évoqués, soit des réglementations imposées par les administrations gouvernementales ou régionales, ou encore des contraintes économiques internationales qui bouleversent les modalités internes de la gestion locale, etc. ; et, à leur suite, les effets locaux de retour face à ces réglementations et contraintes, qui se manifestent par des actions d'opposition ou de restructuration, dirigées à la fois vers l'intérieur et vers l'extérieur, et par bien d'autres effets réciproques de ces dynamiques croisées entre le local, le régional, le national et l'international.

\section{Les approches}

Toutes ces données ne nous ont été accessibles que par des investigations patientes auprès des habitants de Sasaguri et dans les archives locales. Nous sommes tous partis de l'enquête menée collectivement durant sept ans, au rythme d'au moins une semaine à environ un mois sur place chaque année. Dès 2005 , chacun d'entre nous avait à peu près défini l'angle sous lequel il pensait aborder la thématique globale. Néanmoins, nous avons tous continué à procéder à des investigations plus larges que celles qui étaient exigées par ces approches individuelles. Les principaux axes de l'enquête furent l'organisation de la vie sociale et familiale, le fait religieux coutumier, le nouveau pèlerinage de Shikoku à Sasaguri, le shugendō, les ritualités collectives, l'histoire des femmes, les rapports économiques et sociaux à l'environnement et la gestion des ressources naturelles. Dans le même temps, nous avons exploré toutes les ressources historiques et administrativcs acccssibles par les archives et les services communaux. Le temps passé à Sasaguri a donc été partagé entre les démarches auprès des habitants, dans les villages, le centre de la commune, et la recherche puis le dépouillement de toutes sortes de documents. En plus des séminaires de recherche qui ont jalonné nos séjours à Sasaguri, chaque soir nous mettions en commun nos observations et questionnements du jour. Ceci permit à tous de bénéficier des collectes de chacun. Il faut ici noter que le fait d'être revenus pendant sept ans consécutifs dans la commune a été capital, tant pour notre compréhension de la vie locale que pour notre insertion parmi ses habitants. Le temps nous a ouvert quantité de portes - chez les habitants, mais aussi dans notre propre appréhension des choses - qu'un seul long séjour n'aurait jamais permis. Ainsi sommes-nous devenus en quelque sorte des " étrangers " de "l'intérieur ».

Les huit contributions de ce volume représentent chacune une étude sur le thème choisi. Mais elles reposent toutes sur le travail commun et renvoient les unes aux autres pour un meilleur approfondissement des contenus. À ce titre, si chacune d'elles forme un tout, elles sont néanmoins toutes étroitement liées et conçues pour faire sens ensemble, et donc être publiées ensemble. Des notes signalent où trouver les apports complémentaires sur certains points précis dans les autres contributions. Chaque contribution resitue son propos dans le cadre des études et des débats actuels sur la question, en ethnologie du Japon, en anthropologie sociale, et il est 
donné un rapide aperçu de l'état de ces questions. Le cadre social et historique est lui aussi explicité.

Les deux premières contributions posent le cadre socioculturel, historique et environnemental du volume.

Dans «Continuités et transformations de la société locale : le fait coutumier dans le village de Wakasugi », Suzuki Masataka 鈴木正崇 concentre son propos sur Wakasugi, l'un des anciens villages, d'abord intégré en I889 à Seto, puis à la commune de Sasaguri en 1955, avec toutes les autres composantes de Seto. Situé sur le flanc nord du mont Wakasugi, ce village a conservé jusqu'à nos jours une forte identité communautaire fondée sur une longue histoire socioculturelle, religieuse, politique, ainsi que sur des pratiques, un discours et des représentations qui lui sont propres, le tout inscrit dans le cadre distinctif de cette montagne. L'auteur a pris pour focale le fait religieux coutumier à travers lequel il analyse l'organisation sociale, les pratiques et le système de représentations. Les grandes ruptures modernes (fin Edo, Meiji, les guerres du xx ${ }^{e}$ siècle, l'après-guerre, aujourd'hui) sont les articulations par lesquelles il cherche à mettre en lumière le fonctionnement des structures locales au moment de leur destruction et de leurs restructurations successives. Pour ce faire, il privilégie trois niveaux d'expression : le dire, le faire et l'être dans la société. Est pris comme point de départ l'ensemble des histoires des origines (engi 縁起) du mont Wakasugi et de ses structures de culte mixtes (une communauté bouddhique avec des pratiquants du shugendō à l'époque ancienne ; un sanctuaire administré par un yamabushi-desservant des dieux affilié au shugen du mont Hōman 宝満山 à l'époque d'Edo ; un sanctuaire desservi par un prêtre shintō à partir de Meiji). Les éléments de ces récits analysés en premier lieu servent ensuite, tout au long de l'étude, de trame et de jalons pour éclairer la cohésion de l'ensemble local. Cette analyse première, qui démantèle une série de narratifs en éléments distincts, permet de faire d'abord apparaître leur caractère disparate. Dans un second mouvement, la démarche de l'auteur sera justement de montrer comment tous ces éléments « tiennent ensemble », non seulement par le fait du discours narratif, mais surtout par la façon dont ils sont utilisés, dans le vécu du groupe local, aux points cruciaux des structures et des fonctionnalités. Ces narratifs - le dire, ou le dit de Wakasugi - témoignent en effet de réorganisations successives d'éléments discursifs, qui, selon les époques, les auteurs et donc les intentions, sont articulés différemment et sont porteurs de significations différentes. Or ces histoires sont essentielles puisqu'elles vont par la suite être convoquées à toutes époques pour légitimer des actes et des positions internes au village face à toutes sortes d'extériorités. L'exposé de tous ces noms et histoires diverses est donc nécessaire à la compréhension. Tous les éléments qui y sont présentés sont ensuite explicités lors de l'examen des pratiques à l'intérieur desquelles ils jouent un rôle-clé. La lecture permet en effet d'éclaircir progressivement non seulement les contenus réels, mais aussi et surtout la façon dont ceux-ci sont organisés ensemble et constituent une totalité signifiante pour les habitants de Wakasugi. Ce dire créé localement est le noyau des représentations du dedans. Il exprime la façon dont les habitants envisagent la relation de tous ces éléments entre eux, c'est-à-dire en quoi leur agencement constitue «leur histoire », en tant que construction sans cesse continuée. C'est par ce biais que pratiques religieuses 
et sociales - le faire - et organisations - l'être social — sont ensuite analysées. Dans ce but, l'auteur met en perspective l'intérieur et l'extérieur du village selon plusieurs échelles : spatiales — Sasaguri, le canton de Kasuya, la région nord de Kyūshū, tout Kyūshū, l'ouest du Japon, le pays, la Nation et l'étranger - et temporelles. Le recours aux données historiques est utilisé ici comme un instrument de décryptage de l'imbrication complexe de tous ces éléments. C'est ainsi que les liens entre tous les éléments présentés au début émergent au fur et à mesure qu'est analysé le fonctionnement de ce groupe villageois. Activités, actes individuels et collectifs tissent entre eux le maillage qui les relie sans cesse les uns aux autres. On y voit en particulier l'impact décisif des politiques féodales, régionales puis nationales sur les activités de production, les ritualités, les cultes, les structures religieuses et sociales de cette communauté locale, ainsi que la façon dont les habitants ont géré stratégiquement ces politiques. Wakasugi est sans doute une communauté locale qui tranche par la continuité de sa solidarité inscrite dans un contexte environnemental fort. En cela, cet exemple permet de mieux apercevoir des dynamiques qui sont moins nettes ailleurs. L'importante bibliographie fait aussi de cette contribution une source documentaire sur l'histoire religieuse de la région.

Anne Bouchy, dans « Les rapports communautaires aux espaces forestiers entre politiques du dehors et stratégies du dedans : les montagnes-forêts de Sasaguri », aborde la question des rapports communautaires à l'environnement en s'intéressant plus particulièrement aux liens tissés avec les espaces forestiers de Sasaguri. Les relations et les conflits entre système juridique, droit coutumier, histoire économique et gestion des forêts sont placés au cœur de la problématique. L'examen de ces espaces, les montagnes-forêts, qui semblent former une continuité végétale, fait en effet apparaître la multiplicité non seulement des milieux forestiers eux-mêmes, mais également celle des organisations qui les gèrent. D'une part, cette multiplicité physique se révèle le produit d'une histoire locale des rapports aux forêts, dépendant elle-même de politiques régionales, nationales et internationales. D'autre part, l'élément partagé dans cette diversité est la gestion communautaire et son importance. Celle-ci a néanmoins pris des formes diverses selon les époques et les groupes locaux : communaux, coopératives, forêts à bénéfices partagés. Aujourd'hui, certaines de ces formes - notamment les communaux - sont réévaluées tant par les forestiers, par les habitants que par les chercheurs en sciences humaines qui y voient l'un des piliers du vivre ensemble - comme l'avait bien vu le gouvernement de Meiji qui chercha à les éliminer dans la dynamique de la construction de l'État-nation. La question de la «montagne villageoise ", sato-yama 里山, qui a émergé récemment sur le devant de la scène scientifique, politique et culturelle, est resituée ici dans le contexte de ses usages historiques et de son évolution contemporaine à l'échelle locale. L'étude montre ainsi la part déterminante du social dans les relations à une diversité végétale complètement anthropisée, qui n'a cessée d'être transformée, maîtrisée et parfois détruite par les habitants. Ceci notamment parce qu'eux-mêmes sont placés au point de rencontre de leurs besoins et des contraintes imposées par ces milieux ainsi que par quantité d'impératifs extérieurs. L'analyse montre en quoi l'histoire des législations, des régimes de propriété et des restructurations administratives des communes joue un rôle majeur dans les recompositions locales qui vont tantôt 
dans leur sens ou tantôt contre elles. L'auteur a tenté de réunir les approches des milieux végétaux, des organisations sociales et du religieux, et de voir en quoi ce dernier a pu jouer un rôle déterminant dans la sauvegarde des forêts. Aujourd'hui, ces milieux forestiers font face à un nouveau tournant. Et plutôt que sur l'exploitation du bois actuellement en perte de vitesse, leur avenir semble reposer sur la rencontre des nécessités et possibilités internes à la commune et des besoins des citadins qui viennent y pratiquer la «thérapie forestière ».

Les quatre contributions suivantes traitent chacune sous un angle particulier des « textures du religieux » à Sasaguri.

Mori Hiroko 森弘子, dans « Le mont Wakasugi, son sanctuaire et le shugen dans l'histoire de Kyūshū et de l'Asie de l'Est ", resitue finement le maillage dans lequel s'insère localement et extra-régionalement (capitale, centres shugen régionaux et nationaux, continent asiatique) l'histoire du culte du sanctuaire Taiso 太祖神社 qui se dresse sur le sommet du mont Wakasugi. Pour cela, elle s'intéresse aux réseaux et aux dynamiques transversales reliant à toute la région et même au reste du pays le mont Wakasugi, siège d'une entité religieuse qui dépasse les limites communales. Cette dernière est en effet constituée de deux faces, à savoir les deux organisations cultuelles et villageoises situées sur chacun des flancs nord (Utani 右谷) et sud (Satani 左谷) de la montagne. Celles-ci sont elles-mêmes reliées par leur histoire à des ensembles plus vastes. Sont ainsi éclairés les multiples liens que ce sanctuaire entretient avec le culte de Hachiman 八幡, divinité omniprésente à Kyūshū, toujours associé à celui de l'impératrice Jingū 神功皇后, comme c'est le cas ici, et donc également avec un grand nombre de sanctuaires de toute la région septentrionale de Kyūshū. L'analyse met en évidence la façon dont les discours explicatifs des différentes histoires des origines ne cessent de renvoyer l'un à l'autre dans une quête d'identification à une seule et même entité-puissance, qui dépasse les cultes locaux pour les unifier en une figure plus universelle, dont l'idée a été véhiculée par l'enseignement bouddhique. Tous ces liens ont pour arrière-plan des réseaux de pouvoir religieux, économiques et politiques influents. L'autre réseau d'insertion est celui du shugendō, sur lequel Wakasugi offre des documents abondants et totalement inédits qui sont présentés ici. Ce réseau est celui du shugen des monts Hōman et Hiko du nord de Kyūshū, et celui de Kumano 熊野, puis de la branche Honzan 本山派 dont l'échelle est nationale. Aussi les clés de compréhension du shugen du mont Wakasugi sont-elles recherchées dans les dynamiques qui traversent à la fois les cultes locaux des dieux du sol (Taiso, Hachiman, Hōman), le réseau du shugen régional (Hōman et Hiko) et le shugen de Kumano à l'échelle nationale. L'auteur montre comment l'ensemble est réuni d'un côté par la mer et les relations maritimes à la fois nationales (intra-archipel) et internationales (la mer constituant un lien avec le continent asiatique), et de l'autre, par les voies terrestres, notamment celles des entrées dans la montagne, le parcours rituel des groupes shugen. Les dieux et les entités bouddhiques au Japon ont une identité qui est déterminée par l'histoire de la fondation, ou l'implantation, le transfert du culte et de ses structures, et donc par l'histoire de ceux qui gèrent financièrement, politiquement et rituellement ces structures. À ce titre, dieux et bouddhas, leurs officiants et les structures gérées par ces derniers sont un miroir de l'histoire sociale locale. Mori Hiroko montre la complexité des imbrications de tous 
ces paramètres à l'échelle d'un sanctuaire, de son environnement local et régional, et resitue ceux-ci dans le cadre plus large de l'histoire nationale et des relations entretenues par cette région très particulière de Fukuoka avec le continent asiatique, notamment avec la péninsule coréenne et la Chine.

En abordant la question du pèlerinage sous l'angle des dynamiques du dedans et du dehors, Nakayama Kazuhisa 中山和久 a voulu en renouveler l'approche. Dans son étude « La dynamique de création, réplication et déclin des lieux de pèlerinage : le nouveau pèlerinage de Shikoku à Sasaguri ", il fait d'abord une présentation très complète et inédite de l'histoire et des réalités contemporaines de ce pèlerinage, qui reproduit dans l'espace communal le circuit des 88 temples de Shikoku centré sur le culte du Grand Maître (Kōbō daishi 弘法大師, c'est-à-dire Kūkai 空海). Puis il analyse le cas paradigmatique de Sasaguri qui se trouve précisément au croisement de ces dynamiques tant en ce qui concerne sa création, que sa continuité ou son déclin. Pour cela, il met en évidence cinq types de dynamiques, celles de création de l'objet de pèlerinage, de réplication, de genèse du pèlerin, de transformation du pèlerin par les lieux de pèlerinage et celle de transformation des lieux de pèlerinage par les pèlerins. À chaque étape est ainsi montrée l'existence déterminante et nécessaire d'une convergence de l'intérieur et de l'extérieur. Il a fallu pour ce faire des influences externes, des démarches d'individus isolés mais charismatiques, comme la nonne Jinin 慈忍, fondatrice du pèlerinage venue de l'extérieur, et aussi les vogues successives du culte du Grand Maître à l'échelle nationale et régionale, ou encore l'attente des pèlerins. Mais ont été également non moins indispensables les besoins, la demande et les possibilités (économiques, spatiales, politiques) des habitants de Sasaguri ainsi que leurs actes (création du circuit des 88 statues, combat pour sauver le pèlerinage de l'interdiction à Meiji). C'est précisément cette conjonction qui crée le pouvoir d'attraction d'un circuit de pèlerinage. Le principe de la réplication au fondement même de ce pèlerinage est au cœur de la question et éclaire la thématique commune de ce volume. Or ce principe repose sur un jeu entre des ressources de puissance subtile $^{26}$, ou de pouvoir, qui se trouvent à l'extérieur du territoire où le pèlerinage va être implanté, et qui sont considérées comme pouvant être reproduites, transportées, et intégrées avec toute leur efficacité à l'intérieur d'un milieu et d'un groupe autre que celui d'origine. L'auteur signale ici l'importance de la transformation du dedans par le détournement de modèles du dehors. Il souligne que cela doit attirer l'attention sur les faits de sortie des pratiques coutumières vers l'extérieur des groupes qui les ont produites. Une telle problématique a peu fait l'objet de recherches en ethnologie du Japon, qui a eu tendance à privilégier le regard sur ce qui se passe à l'intérieur des communautés locales. Il fait également ressortir le fait que, dans l'examen des forces externes et internes en présence, on ne doit pas seulement tenir compte de celles qui sont d'ordre factuel ou matériel, mais aussi de celles qui viennent du cœur.

Une seconde étude de Suzuki Masataka, intitulée «Modernisation des temples bouddhiques et société locale : le Nanzō-in de Sasaguri » aborde la question du nouveau pèlerinage de Shikoku à Sasaguri sous un angle opposé à celui qui est adopté

26. Principe agissant ou « efficace 》 (reiryoku 霊力 ou reigen 霊験) des entités spirituelles qui peut aussi être détenu par des humains. 
par Nakayama Kazuhisa, dans la contribution précédente. C'est-à-dire, non plus à partir de sa totalité, mais de son centre, le Nanzō-in. Or le cas du Nanzō-in est ici particulièrement riche pour notre réflexion. Ce temple a été introduit à Sasaguri, à Meiji, à la demande des habitants, afin de contrecarrer la décision du gouvernement d'interdire ce pèlerinage. Car celui-ci était originellement constitué d'un circuit reliant entre eux 88 simples oratoires à la garde des habitants, et n'avait donc aucun lieu de culte institutionnel. La version officielle veut que le Nanzō-in ait été d'abord un temple du mont Kōya, centre par excellence du culte du Grand Maître (Kōbō daishi), d'où il aurait été transféré à Sasaguri à cette époque. C'est donc une structure de l'extérieur qui a sauvé le pèlerinage de la destruction. L'auteur met en lumière les processus par lesquels ce temple, grâce à l'action de ses trois supérieurs (trois générations successives de la même famille), est devenu une institution du «dedans » à part entière, essentielle à la vie de toute la commune de Sasaguri. Le principe de ce succès repose sur une très inventive capacité de ces supérieurs à répondre à la demande, aussi bien des pèlerins de l'extérieur, que des fidèles de l'intérieur et de l'extérieur, en conciliant religion, commerce et médiatisation. Parfois surprenante, la mise en œuvre conjuguée de tels moyens s'est révélée parfaitement efficace pour la conservation de la connaissance du passé et l'adaptation à la marche du monde. On voit ainsi, au cours du $\mathrm{Xx}^{e}$ siècle, les activités du temple se déployer dans de nombreuses directions, non seulement religieuses mais aussi commerciales et médiatiques. Tout en étant une force locale de premier plan, le temple dépend lui-même beaucoup de l'impact de ses stratégies économico-religieuses sur l'extérieur de la commune. Si cela leur vaut des critiques d'ordre divers, le Nanzō-in et ses supérieurs représentent une tendance novatrice du religieux, qui, tôt ou tard, finit par inspirer ou même servir de modèle aux formes plus établies lorsque celles-ci sont en perte de vitesse. L'étude montre combien toutes ces dynamiques sont d'une grande actualité et animent des pratiques en pleine vitalité qui vont en s'enrichissant constamment.

Avec « La pierre qui vit : naissance et mort des statues dans une ville de pèlerinage ", Charlotte Lamotte s'introduit encore plus au centre de l'intériorité, puisqu'elle aborde la dimension de l'individu et de sa relation au religieux. Le médiateur entre l'intérieur et l'extérieur est ici la statue, objet de vénération. En partant d'une riche ethnographie faite sur le circuit du nouveau pèlerinage de Shikoku à Sasaguri, elle s'interroge sur les « interactions » qui s'établissent entre fidèles et objets de culte. Selon cette analyse, un triple schéma se dessine. Une vie est donnée à la statue par le rite d'animation qui lui confère une âme (mouvement de l'extérieur vers l'intérieur) ; puis les attentions qui lui sont portées par le fidèle, les intentions (de celui-ci) dont elle devient le dépositaire et les "réponses " qu'elle est censée lui adresser entretiennent une « vie » de l'objet cultuel (en un mouvement double, dans les deux sens). Enfin, ce processus conduirait à la fusion de deux « intériorités », celle du fidèle et celle de la statue, cette dernière étant le résultat de la projection de toutes les intentions du fidèle qui a fait vou de s'en occuper. La médiation entre ces deux intériorités est ici faite par l'âme. Telle est la théorie locale de la figuration et des effets du culte qui se dessine ici. Dans ce cadrc d'analyse anthropologique, les dynamiques internes et externes apparaissent bien comme ce qui construit et définit les entités et les personnes, tout comme le contour des intériorités et des extériorités. 
Les deux dernières contributions se penchent sur la vie des maisons et celle des femmes de Sasaguri.

Ishikawa Toshiko 不川稔子, dans «Les rites annuels de la maison et des communautés locales en transformation : intérieur et extérieur des lieux de vie communautaires ", fait une analyse méticuleuse des bouleversements qui, au Xx siècle et encore aujourd'hui, ont marqué et marquent le domaine intime de la maison, entendue ici comme le groupe familial vivant sous un même toit depuis plusieurs générations. Pour ce faire, elle choisit d'examiner les transformations des ritualités à partir de la logique du lieu, des acteurs et des finalités. Deux niveaux sont abordés, les relations entre l'intérieur et l'extérieur de la maison d'habitation pour une même famille, et les rapports entre maison et communauté locale tels qu'ils s'articulent autour du lieu lors des rites collectifs et des fêtes annuelles. Elle pose ainsi deux pôles d'intériorité, la maison et la communauté locale restreinte, entre lesquels les ritualités bougent et passent tantôt de l'intérieur à l'extérieur des lieux et des groupes, selon des dynamiques liées elles-mêmes au mouvement de l'histoire locale et globale. Grâce à de nombreux exemples très précis et finement analysés, on peut ainsi voir se dessiner les processus de transformation drastique du caractère des lieux sous l'impact des modifications matérielles des modes de vie, et des bouleversements touchant l'économie de subsistance et les acteurs rituels eux-mêmes. L'analyse se porte notamment sur les changements de la place et du rôle rempli par les femmes - situées au cœur du "dedans » - en tant qu'acteurs rituels venant remplacer les hommes, et aussi sur la valeur identitaire du rôle rituel féminin. Selon le contexte, l'extériorisation des rites peut avoir un effet positif ou négatif sur leur continuité. Partant d'une présentation très précise des changements les plus actuels des ritualités familiales et communautaires (tant à l'échelle du quartier, qu'à celle des groupes d'âge ou des confréries), cctte contribution attire l'attention sur le rôle déterminant des rites, non seulement pour la construction des entités familiales et collectives, mais aussi pour la pérennité de tout un héritage de transmissions orales qui leur sont liées et constituent l'un des fondements de l'identité locale. Elle pose ainsi la question de la maison prise entre modèle(s) et réalités fort diverses dans la société contemporaine.

Kanda Yoriko 神田より子 adopte une posture tout à fait particulière dans son approche des femmes de Sasaguri : "Femmes de Sasaguri : au fond du religieux et à l'ubac du séculier ". Sur le modèle de l'« ethnologue qui marche ", Miyamoto Tsune. ichi 宮本常- (1907-1981), elle privilégie en effet la narration directe des femmes qu'elle a rencontrées, en nous retransmettant leurs paroles à travers lesquelles s'expriment les relations et les articulations entre intériorité et extériorité sociales. Il s'agit d'une ethnographie narrative, qu'elle propose comme méthode performante pour recueillir ce que personne ne dit à l'extérieur, et pour mieux entrer dans l'intimité des locuteurs. Les limites de cette publication ne permettant pas de retranscrire la totalité des témoignages directs, une partie en a été résumée par l'auteur. Mais l'ensemble fera partie de la base de données de Sasaguri, qu'il est prévu d'ouvrir sur le site de l'EFEO. La contribution suit donc le fil du discours de femmes épouses de spécialistes du religieux ou qui les côtoient, ou encore qui sont spécialistes religieux elles-mêmes, celui de femmes laïques membres de confréries pour le pèlerinage à Ise 伊勢, et aussi celui de femmes qui ont travaillé dans les mines de Sasaguri ou à 
leur ombre comme prostituées. Tous ces témoignages permettent à l'auteur de questionner l'intériorité et l'extériorité de ces femmes selon la position qu'elles occupent à un moment ou à un autre. Ainsi autour de l'axe religieux/sacré - laïque/profane émerge toute une gamme de positions extérieures et intérieures, qui se révèlent en fait changeantes chez un même individu/sujet selon le moment, le lieu, le type d'action envisagée, et qui s'entrecroisent entre elles et avec celles des différents protagonistes de tous les espaces-temps concernés. On voit alors clairement combien les notions de dedans et dehors sont à la fois relatives et modulables.

L'anonymat des interlocuteurs a été respecté dans certains cas, mais dans d'autres, lorsque la connaissance du nom de la personne était déterminante pour le sens des propos, les locuteurs ont été identifiés, et ce, avec leur accord.

Nous avons souhaité ajouter un complément original à ce volume. En effet, le contenu de cette publication ne représente qu'une partie de la totalité des données collectées et des connaissances acquises. Il est donc prévu que soit ouverte prochainement sur le site de l'EFEO une « base de données Sasaguri », qui comprendra des fiches individuelles sur des points précis, des documents originaux, des photos, ainsi que le texte des contributions en japonais. Ceci offrira ainsi aux lecteurs occidentaux la possibilité de consulter les textes japonais pour rechercher par exemple des caractères non intégrés dans les traductions, et aux lecteurs japonisants de lire les textes dans leur totalité. À cause des limitations éditoriales, la traduction française a dû condenser ou abréger certains passages avec l'accord des auteurs.

$\mathrm{Si}$, dans certaines contributions, le début reprend plus ou moins une présentation de la commune indispensable à la bonne intelligence des logiques que chacune d'entre elles cherche à mettre en lumière, c'est parce que nous avons pensé qu'il fallait aussi envisager la possibilité de la lecture et de l'utilisation de chacune d'elles indépendamment et de toutes les autres et de la présentation du volume. Deux cadres principaux sont en effet susceptibles d'un tel usage : la base de données informatisées, prochainement disponible sur le site de l'EFEO, réunissant ces études avec les matériaux ethnographiques, et le cadre pédagogique dans lequel celles-ci peuvent être exploitées pour l'enseignement et la formation à la recherche, notamment en ethnologie du Japon. La redondance qui en résulte quelque peu à l'échelle du volume doit être comprise comme une ouverture vers une plus large diffusion de chaque texte.

À l'exception de quelques-unes, toutes les photographies ont été prises par les auteurs.

Comme complément aux références bibliographiques de chaque contribution, est aussi insérée une bibliographie commentée d'ouvrages de référence dans les principaux champs de l'ethnologie du Japon, notamment de ceux qui se rapportent aux thématiques de cet ouvrage. En dehors de quelques textes fondamentaux un peu anciens, le choix s'est porté sur les études récentes.

À l'issue du long cheminement qui a abouti à la rédaction et à la publication de ce volume, nous voudrions remercier tous les habitants des vingt-et-un quartiers de Sasaguri, tous les supérieurs et les moines des temples du pèlerinage et de la commune, les desservants des sanctuaires, pour leur accueil et leur coopération, sans lesquels ce travail collectif n'aurait pu voir le jour. Nous gardons en mémoire tous 
ces moments de dialogue qui se sont déroulés chez eux, sur le bord des chemins et des rizières, sous le couvert des forêts de cryptomères, dans les temples et les oratoires, ou encore lors des fêtes et des occasions rituelles. Il n'est pas possible de citer le nom de chacun d'eux ici, car la liste en serait beaucoup trop longue. Mais nous voulons leur adresser, à toutes et à tous, notre plus sincère reconnaissance.

Nos remerciements et notre reconnaissance vont également à Miura Tadashi 三浦正, maire de Sasaguri, qui nous a d'emblée encouragés et soutenus tout au long de notre entreprise, ainsi qu'à ses adjoints, aux conseillers municipaux et à tous les responsables des services de la mairie qui ont toujours accueilli chaleureusement nos démarches répétées et souvent intempestives. Notre reconnaissance et notre amitié vont aussi tout particulièrement vers Kurose Shigefumi 黒瀬茂文, qui, en tant que responsable du Centre de documents historiques et de matériaux ethnographiques de Sasaguri, a été un véritable guide tout au long de ces sept années, tant par son aide logistique et intellectuelle que par sa présence amicale; et aussi à ses assistants Kurokawa Takashi 黒川隆志, Moribe Miki 森部美紀 et Kinugasa Junko 衣笠順子, dont l'infatigable soutien nous a permis d'accéder à d'innombrables documents, et au cour des montagnes-forêts. Nous remercions aussi la famille Murashima 村島 ご一家, qui nous a accueillis avec cour durant toutes ces années dans son auberge Ebisuya 恵比寿屋旅館, devenue pour nous au fil du temps une véritable maison, ainsi que Kominami Mitsuyo 小南満代, qui nous a également prêté sa demeure familiale.

Notre reconnaissance va également à tous les chercheurs qui, lors des séminaires de recherche à Sasaguri, nous ont apporté leur aide pour mieux comprendre les données locales dans le contexte régional de Kyūshū, et parmi eux, tout particulièrement à Fukuma Yũji 福間裕爾, Hisano Takashi 久野隆志, Ino.ue Susumu 井上晋, Ishitaki Toyomi 石瀧豊美, Mutō Gun.ichirō 武藤軍一郎, Namihira Emiko 波平恵美子, Sasaki Tetsuya 佐々木哲哉, Shirakawa Takuma 白川琢格, Yoshidome Tōru 吉留徹.

Enfin, nous remercions l'EFEO et la Fondation du Japon pour leur soutien financier sans lequel ce projet n'aurait pu être mené à bien, ainsi que Benoît Jacquet et Iyanaga Nobumi 彌永信美, respectivement responsables des centres EFEO de Kyōto et Tōkyō, pour leur méticuleux travail d'édition de ce volume des Cabiers d'Extrême-Asie, ainsi que pour leur chaleureuse amitié, et aussi Astrid Aschehoug et Chloé Beaucamp du service des éditions de l'EFEO à Paris pour leur efficace collaboration lors de la finalisation du volume. 